\title{
Ghost Resonance in a Semiconductor Laser with Optical Feedback
}

\author{
J.M. Buldú ${ }^{1}$, Dante R. Chialvo ${ }^{2,3,4}$, Claudio R. Mirasso $^{2}$, M.C. Torrent ${ }^{1}$, and J. García-Ojalvo, ${ }^{1}$ \\ ${ }^{1}$ Departament de Física i Enginyeria Nuclear, Universitat Politècnica de Catalunya, Colom 11, E-08222 Terrassa, Spain \\ ${ }^{2}$ Departament de Física, Universitat de les Illes Balears, E-07071 Palma de Mallorca, Spain \\ ${ }^{3}$ Department of Physiology, Northwestern University, Chicago, Illinois, 60611, USA \\ ${ }^{4}$ Center for Studies in Physics and Biology, The Rockefeller University, New York, New York 10021, USA
}

\begin{abstract}
We show both experimentally and numerically a ghost resonance in the sudden power dropouts exhibited by a semiconductor laser subject to optical feedback driven by two simultaneous weak periodic signals. The small signal modulation conspires with the complex internal dynamics of the system to produce a resonance at a ghost frequency, i.e. a frequency that is not present in the driving signals. This is an eminently nonlinear effect not reported before and agrees with the recent theoretical predictions by Chialvo et al. [Phys. Rev. E 56, 050902(R), 2002].
\end{abstract}

Semiconductor lasers subject to optical feedback have attracted the attention of the researchers for more than three decades. One of the most interesting regimes that these systems exhibit is the Low-Frequency Fluctuation regime (LFF) in which the output power of the laser suffers sudden dropouts to almost zero power at irregular time intervals when is biased close to threshold [1]. Although the LFF behavior has been observed already at the end of the seventies, its dynamics is not fully understood yet.

Recent experimental [2] and numerical [3,4] reports show the conditions for which a laser subject to optical feedback and biased close to threshold is able to operate in an excitable regime, before the onset of the LFFs. This means that a laser prepared in such a state is stable under small periodic perturbation of the bias current and exhibit the three ingredients of any excitable system, namely: the existence of a threshold for the perturbation amplitude above which the dropout event can occur; the form and size of the dropout events are invariant to changes in the magnitude of the perturbation, although multipulse emission has been also predicted [5]; a refractory time exists: if a second perturbation is applied at a time shorter than the refractory time, the system no longer responds.

It has been also shown both experimentally [6,7] and numerically $[8,9]$ that a laser subject to optical feedback can also exhibit stochastic [10] and coherence [11] resonance when biased close to threshold, extending the richness of the dynamical behaviors of this system. Stochastic resonance is characterized by an optimum coherence of the system output with a weak periodic signal for an intermediate value of the noise level. On the other hand, coherence resonance is characterized by an almost periodic response of the system to an intermediate level of noise but without any external periodic signal. Both effects have been also observed in a large variety of systems including periodic and chaotic systems $[10,12]$.

Also recently it has been shown that the laser responses can be entrained to give a periodic train of dropouts by superimposing an external forcing to a bias current close to threshold. If the amplitude of the forcing is larger than a certain value, the dropouts occur at the frequency of the external forcing when the latter has a frequency larger than the mean frequency of the dropouts in the absence of the perturbation [13-15].

In all the previous studies, semiconductor lasers were excited at most with a single sinusoidal input. In this letter we go further and study experimentally and numerically the response of a semiconductor laser subject to optical feedback biased close to threshold modulated by two weak sinusoidal signals. The main point of our results is that the system response shows a resonance at a frequency that is absent in the input signals. For that reason we term it ghost resonance. We describe the condition for and the location of this ghost resonant frequency which has been recently predicted, for a simpler system, by theoretical arguments in ref. [16].

The experimental setup, shown in Fig. 1, consists of an index-guided AlGaInP semiconductor laser (Roithner RLT6505G), with a nominal wavelength of $658 \mathrm{~nm}$. The threshold current is $I_{\mathrm{th}}=18.4 \mathrm{~mA}$ for a temperature of $19.86 \pm 0.01{ }^{\circ} \mathrm{C}$. The injection current (IC), without modulation, is set to $19.7 \pm 0.1 \mathrm{~mA}$ all through the experiment. An antireflection-coated laser-diode objective (L) is used to collimate the emitted light. An external mirror (M) is placed $83.5 \mathrm{~cm}$ away from the front facet of the laser, introducing a delay time of $\tau \sim 5.56 \mathrm{~ns}$. The feedback strength is such that it yields a threshold reduction of $7.0 \%$ and it is adjusted by placing a neutral density filter (NDF) in the external cavity. The output intensity is collected by a fast photodetector (PD) and analyzed with a $500 \mathrm{MHz}$ bandwidth acquisition card.

We are interested in the system response to modulation composed of multiple periodic signals $f_{1}, f_{2}, \ldots f_{n}$. Although the present letter focus in the case of two components, the driving signal has the following general form: $I(t)=I_{b}\left\{1+m\left[\sin \left(2 \pi\left(k f_{0} t+\Delta f t\right)\right)+\sin (2 \pi((k+\right.\right.$ 1) $\left.\left.\left.\left.f_{0} t+\Delta f t\right)\right)+\ldots \ldots+\sin \left(2 \pi\left((k+n-1) f_{0} t+\Delta f t\right)\right)\right]\right\}$, with $k>1$ and $n$ being the number of terms used. $I_{b}$ is the bias current and $m$ is the modulation amplitude. Here we choose to use two terms $(n=2)$ and $f_{0}=4.5$ 
$\mathrm{MHz}$ (although the same qualitative features would be observed for other choices of $f_{0}$ ). For simplicity, initially we describe results for $\Delta f=0$, i.e., the singular case of harmonic signals.

In Fig. 2 we show examples of three representative time traces for low $(m=1.05)$, intermediate $(m=1.5)$ and high $(m=2.1)$ amplitude values of the injected signals. It can be clearly seen that for the intermediate amplitude the dropouts are almost equally spaced at a time interval that corresponds grossly to $1 / f_{0}$, (depicted by the double-headed arrow in the middle panel) a frequency that is not being injected. Thus the laser is detecting in a nonlinear way the subharmonic frequency. To better visualize this fact we plot on the right panels the probability distribution functions (PDF) for a large number of dropouts (approx. 1500). For the small amplitude (top-right panel) it can be observed a peak at a time $1 / f_{0}$ and other peaks at longer times which indicate that the system responds sometimes to $f_{0}$ although at some others times dropouts are skipped. For the optimum value of the amplitude (middle-right panel) the PDF has a clear peak at $1 / f_{0}$ which indicates that the system is resonating with this frequency. For the higher amplitude (bottom-right panel) there are several peaks at different times corresponding to higher frequencies.

The resonance with the ghost frequency can be visualized by measuring the mean interval between dropout events and its standard deviation (SD) at various values of the signal amplitude $m$. Fig. 3 shows these results plotted as the normalized SD (i.e., SD/mean) as a function of the mean frequency of dropout events. It is clearly seen that the minimum coincides with the $f_{0}$ (vertical dashed line), i.e., the ghost frequency.

The ghost frequency is not, as one naively would expect, simply the difference between the two components $f_{1}$ and $f_{2}$, (where $f_{1}=2 f_{0}$ and $f_{2}=3 f_{0}$ ). This is demonstrated by adding a small term (i.e., $\Delta f \neq 0$ ) which shifts equally both frequencies. In this case we observe that the resonant frequency shifts as well, despite the fact that the difference remains constant. Results from experimental runs using $f_{1}=7$ to $10.5 \mathrm{MHz}$ and $f_{2}=11.5$ to $15 \mathrm{MHz}$ and selecting the optimum amplitude $m=1.5$ are presented in Fig. 4. The format of the plot is meant to illustrate better the linear change of the resonant frequency $f_{R}$ as a function of the frequency shift. The PDFs are plotted using frequency (i.e., inverse of the dropout intervals) axis and they are lined up with the $f_{1}$ frequency at which were obtained. It can be seen that the density of the most frequent dropouts lies on a straight line. The experimental results show a remarkable agreement with the prediction given in [16] defined by:

$$
f_{R}=f_{0}+\frac{\Delta f}{k+0.5}
$$

Since the range of $f_{1}$ we explored is about twice $f_{0}$, the dotted line labeled ' $\mathrm{k}=2$ ' predicts the location of the most important resonance and the one ' $\mathrm{k}=3$ ' the expected ones if the range where to be extended further up. Thus, the results presented in this figure agree extremely well with the ones described previously in a simpler system in [16] and it is the first experimental demonstration of this type of resonance at the ghost frequency.

We have also checked that our experimental results can be reproduced by the well known Lang-Kobayashi (L-K) model [17], which is the simplest model to describe the dynamics of a semiconductor laser subject to weak/moderate optical feedback. The L-K equations account for single mode operation and describe the time evolution of the slowly varying amplitude of the electric field $\mathrm{E}(\mathrm{t})$ and the excess carrier number $\mathrm{N}(\mathrm{t})$ :

$$
\begin{aligned}
\frac{d E}{d t} & =\frac{1+i \alpha}{2}(G(E, N)-\gamma) E(t) \\
& +\kappa e^{-i \omega \tau} E(t-\tau)+\sqrt{2 \beta N} \xi(t) \\
\frac{d N}{d t} & =I_{b}\left(1+m\left\{\sin \left(2 \pi\left(k f_{0} t+\Delta f t\right)\right)\right.\right. \\
& \left.\left.+\sin \left(2 \pi\left((k+1) f_{0} t+\Delta f t\right)\right)\right\}\right)-\gamma_{e} N(t) \\
& -G(E, N)|E(t)|^{2} \\
G(E, N) & =\frac{g\left(N(t)-N_{0}\right)}{1+s|E(t)|^{2}} .
\end{aligned}
$$

The first term on the right hand side of Eq.(2) accounts for the stimulated emission. $\alpha=3.4$ is the linewidth enhancement factor and $\gamma=0.24 \mathrm{ps}^{-1}$ is the cavity decay rate. The second term is the feedback term which is described by two parameters: the feedback strength $\kappa=20 \mathrm{~ns}^{-1}$ and the external round-trip time $\tau=5.57$ ns. $\omega / 2 \pi=4.56 \times 10^{14} \mathrm{~Hz}$ is the laser free running frequency. The last term accounts for the spontaneous emission noise, considered as a Gaussian white noise source of zero mean and delta correlation, with a spontaneous emission rate $\beta=5 \times 10^{-10} \mathrm{ps}^{-1}$. The first term in Eq.(3) accounts for the injection current with the two sinusoidal inputs at frequencies $2 f_{0}$ and $3 f_{0}$, being $f_{0}=4.5$ $\mathrm{MHz}$ and modulation amplitude $m=1.2$ with respect to threshold. The second term accounts for the spontaneous recombination and the third one for the stimulated recombination. $I_{b}=1.07 I_{t h}$ is the bias current, $I_{t h}=19.8 \mathrm{~mA}$ is the threshold current and $\gamma_{e}=0.62$ $\mathrm{ns}^{-1}$ is the carrier decay rate. Eq.(4) accounts for the material gain which depends linearly on $N$ and slightly nonlinear on $|E|^{2}$. $N_{0}=1.5 \times 10^{8}$ is the number of carriers at transparency and $s=1 \times 10^{-7}$ is the saturation gain coefficient. In Fig. 5 we show the results obtained by numerical simulations presented in the same format as in Fig. 4. It can be clearly seen that qualitative similar results are obtained as in the experiments which indicates that the L-K model is also able to extract the main features of this new resonant phenomenon.

Under the current experimental conditions is cumbersome to change the noise intensity, and thus one is unable to fully explore the stochastic aspects of this resonance as was done in [16]. We find that the most robust results are obtained when the bias is tuned close to the thresh- 
old for LFF, a region where the effects of even minute fluctuations are expected to be magnified. The origin of these fluctuations, whether they are induced by the internal nonlinear dynamics or by stochastic sources remains unclear. The consequences of these aspects deserve to be explored in future work.

In conclusion, we have described, experimentally and numerically, a new type of resonance observed when a semiconductor laser subject to optical feedback is biased close to its excitable dynamics, near the onset of the lowfrequency fluctuation regime. It is shown that when this system is modulated with two weak periodic signals of different frequencies exhibits a resonance at a ghost frequency, i.e., a frequency that it is not present in the modulating input. We find that for injection frequencies $k f_{0}$ and $(k+1) f_{0}$, being $f_{0}$ any slow frequency, we observe the resonance at exactly $f_{0}$, a frequency that is not present in the injection current. It is also observed that when a constant shift is added to both frequencies of the injected signal the resonance does not appear at the difference between the two frequencies but at a frequency that follows a simple linear relationship. Our results confirm the recent theoretical predictions by Chialvo and coworkers, based on a more simpler system [16].

We acknowledge financial support from MCyT (Spain) under projects CONOCE BFM2000-1108, BFM20012159, BFM2002-04369, MCyT and Feder under project SINFIBIO. DRC is grateful for the hospitality and support of the Departmento de Fisica, Universitat de les Illes Balears, Palma de Mallorca, Spain.

[1] Ch. Risch and C. Voumard, J. Appl. Phys. 48, 2083, (1977).

[2] M. Giudici, C. Green, G. Giaconelli, U. Nespolo and J.R. Tredicce, Phys. Rev. E 55, 6414, (1997).

[3] M.C. Eguía, G. Mindlin and M. Giudici, Phys. Rev. E 58, 2636 (1998).

[4] J. Mulet and C.R. Mirasso, Phys. Rev. E 59, 5400 (1999).

[5] S. M. Wieczorek, B. Krauskopf and D. Lenstra, Phys. Rev. Lett. 88, 063901 (2002).

[6] G. Giacomelli, M. Giudici, S. Balle and J.R. Tredicce, Phys. Rev. Lett. 84, 3298 (2000).

[7] F. Marino, M. Giudici, S. Barland and S. Balle, Phys. Rev. Lett. 88, 040601 (2002)

[8] J.M. Buldú, J. García-Ojalvo, C. R. Mirasso, M.C. Torrent and J.M. Sancho, Phys. Rev. E 64, 051109 (2001).

[9] J.M. Buldú, J. García-Ojalvo, C.R. Mirasso, and M.C. Torrent, Phys. Rev. E 66, 021106 (2002).

[10] L. Gammaitoni, P. Hanggi, P. Jung and F. Marchesoni, Rev. Mod. Phys. 70 , 223 (1998).

[11] A. Pikovsky and J. Kurths, Phys. Rev. Lett. 78, 775 (1997).

[12] C. Palenzuela, R. Toral, C.R. Mirasso, O. Calvo and J. Gunton, Europhys. Lett. 56, 347 (2001).
[13] J. Mulet, "Statistics of power dropouts in semiconductor lasers with optical feedback", Master Thesis, Universitat de les Illes Balears, sec. 3.5 (1998).

[14] D.W. Sukow and D.J. Gauthier, IEEE J. of Quantum Electron 36, 175 (2000).

[15] J.M Mendez, R. Laje, M. Giudici, J. Aliaga, and G.B. Mindlin, Phys. Rev. E 63, 066218 (2001)

[16] D.R. Chialvo, O. Calvo, D.L. Gonzalez, O. Piro and G. V. Savino, Phys. Rev. E 56, 050902(R) (2002).

[17] R. Lang and K. Kobayashi, IEEE J. Quantum Electron. 16, 347 (1980).

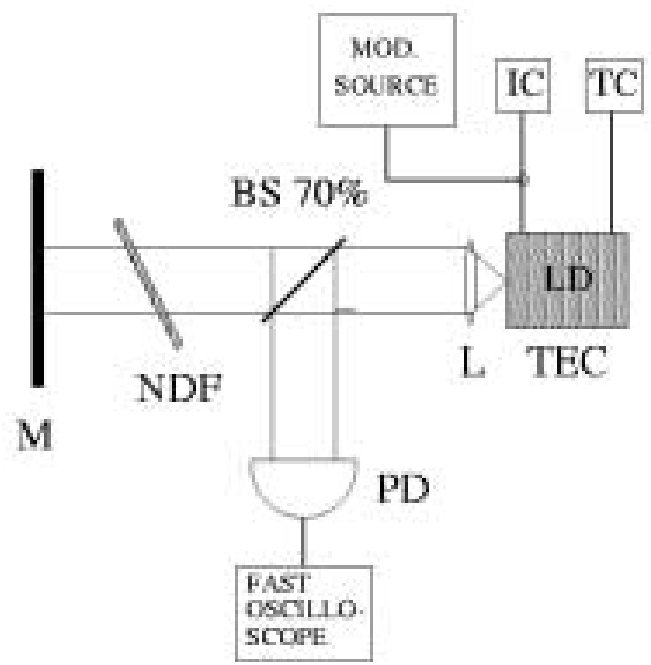

FIG. 1. Diagram of the experimental setup.

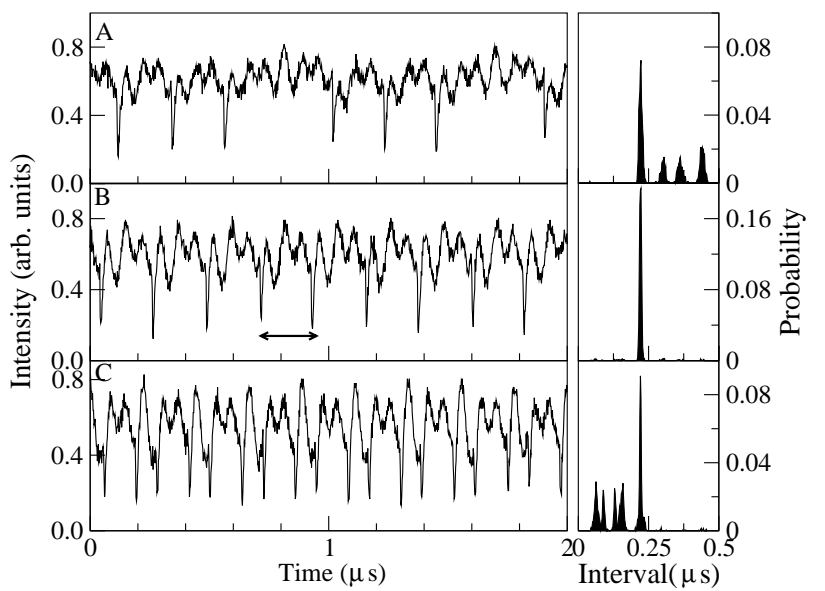

FIG. 2. The left panels show the experimental time series for low (A), medium (B) and high (C) injected signals. The right panels depict the PDFs of the dropouts intervals at the three amplitudes. The PDFs largest peak corresponds to $1 / f_{0}$. 


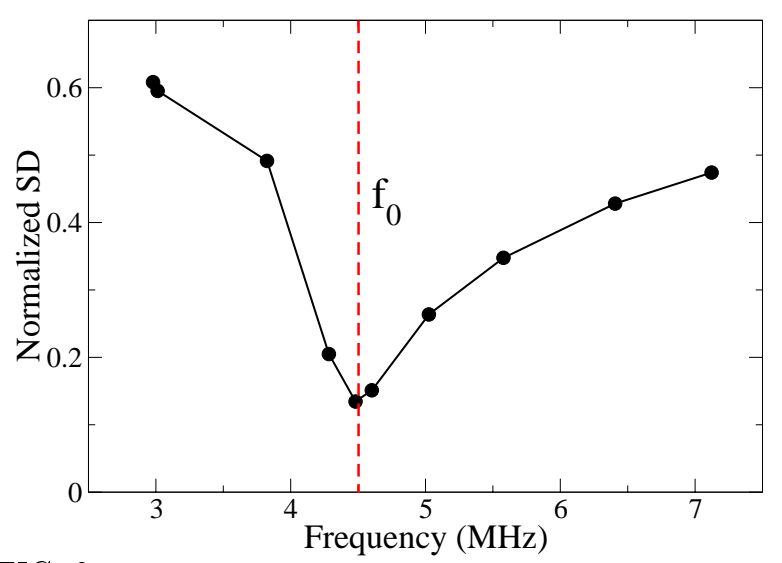

FIG. 3. Experimental results showing that the variability of the dropout intervals reaches a minimum when its frequency approaches $f_{0}$.

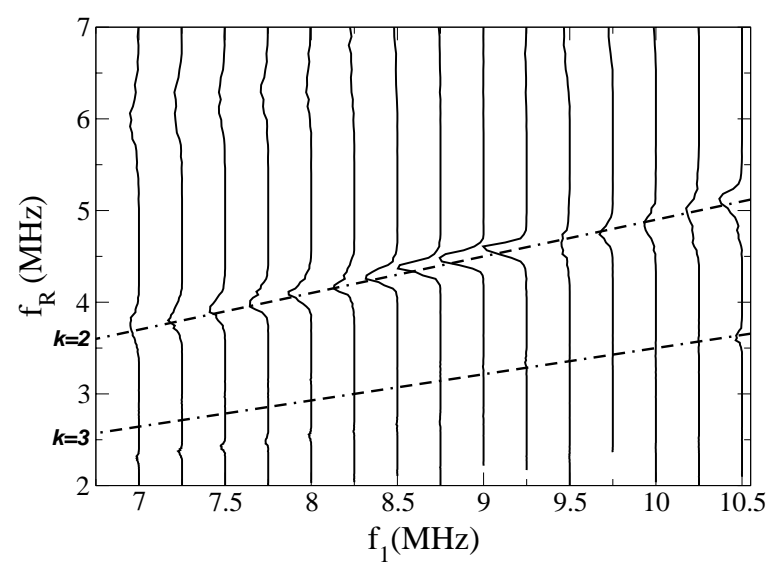

FIG. 4. Experimental results. PDFs of the intervals between dropouts are plotted as their inverse. For each pair of $f_{1}-f_{2}$ frequencies explored the resulting PDF is plotted at the corresponding $f_{1}$ frequency. The lines are expected resonance frequencies from Eq.(1).

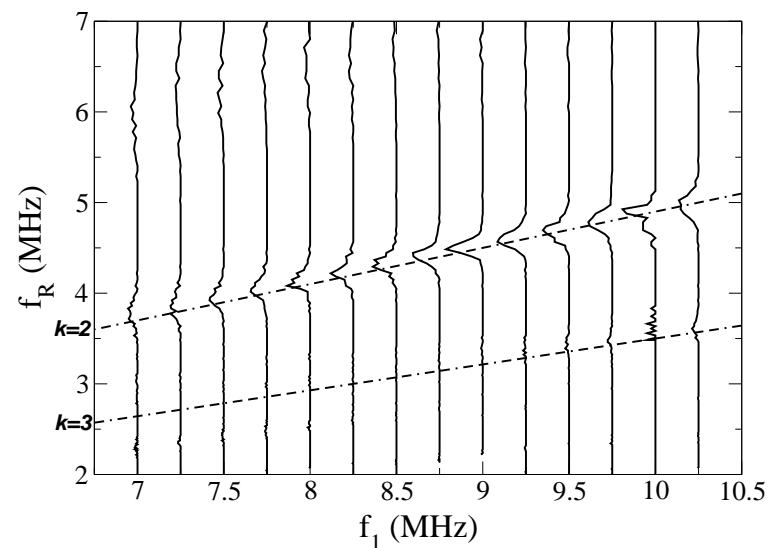

FIG. 5. Numerical results. PDFs of the intervals between dropouts and theoretical lines plotted using the same format as in Fig. 4. 